\title{
Effect of overmolding process on the integrity of electronic circuits
}

\author{
Mona Bakr ${ }^{1}$, Yibo $\mathrm{Su}^{2}$, Frederick Bossuyt ${ }^{1}$, Jan Vanfleteren ${ }^{1}$ \\ ${ }^{1}$ Center for Microsystems Technology, IMEC and Ghent University, Belgium \\ mona.bakr@ugent.be \\ Tel.: +3292646606 \\ 2 Brightlands Materials Center, The Netherlands
}

\begin{abstract}
Traditional injection molding processes have been widely used in the plastic processing industry. It is the major processing technique for converting thermoplastic polymers into complicated $3 D$ parts with the aid of heat and pressure. Next generation of electronic circuits used in different application areas such as automotive, home appliances and medical devices will embed various electronic functionalities in plastic products. In this study, over-molding injection molding (OVM) of electronic components will be examined to insert novel performance in polymer materials. This low-cost manufacturing process offers potential benefits such as, reduction in processing time, higher freedom of design and less energy used when compared to the conventional injection molding method. This paper aims to evaluate the performance of this process and propose a series of alternative solutions to optimize the adhesion between and integration of electronics and engineering plastics. A number of methods are used to optimize the process so that the electronic circuits are not damaged during the overmolding, moreover to test the reliability of the system in order to control the continuity of connections between the electronic circuit foils and the electronic components after the OVM process. Correspondingly, we have performed specific tests for this purpose varying in some conditions: the type of injected plastic used, overmolding parameters (temperature, pressure and injection time), electronic circuit design, type of assembled electronic components, type of foils used, and the effect of using underfill material below the electronic component. From these tests, first conclusions were made. We have also studied adhesion between the foil and the over-molding material. In this case, various types of engineering plastics have been tested; polypropylene $(P P), 30 \%$ weight percentage glass fiber filled polypropylene (GF-PP), Polyamide-6 (PA6) and 50\% weight percentage glass fiber filled polyamide-6 (GF-PA6). It was proved that throughout the wide range of tested materials, (PA6) over-molded samples showed a better adhesion on the copper-polyimide foils than the rest. These plastics were over-molded on two types of polyimide (PI)/Copper $(\mathrm{Cu})$ tracks foils with and without an adhesive layer between PI and Cu. It was obviously clear that the foils with an adhesive layer between PI and $\mathrm{Cu}$ had more delamination in the $\mathrm{Cu}$ tracks than the foils without an adhesive layer. Furthermore, it was shown that the presence of an underfill material has an effect on the system as the foils that had an underfill material below their components successfully had a better connection than the foils without an underfill material. Finally, experiments were executed using the two-probe method as an electrical measurement and microscope investigation as the visual inspection.
\end{abstract}

Keywords: Over-molding process, Flexible foils, Electronic circuits, Engineering plastics, Reliability

\section{Introduction}

Flexible electronic circuits are regularly an alternative for replacing the rigid printed circuit boards (PCBs) in different application areas such as automotive industry, medical devices, and home appliances. They require novel integration methods of electronic functions into products with some benefits such as decreased weight, higher precision, lower costs, reduced operation time and flexible custom design when compared to currently available electronics manufacturing and packaging methods [1]. Thermoset epoxies are the most widely used materials in electronic packaging and printed circuit boards, however, thermoplastics polymers offer superior properties; depending upon their chemistry they can be very much like rubber, or as strong as aluminum. They are organic melt processable materials. This generally means that they are heated, formed then cooled in their final shape. They are environmentally sustainable and have precision in molding capability. In general, the combination of lightweight, high strength, and low processing costs make thermoplastics well suited to many applications. The most common methods of processing thermoplastics are injection molding, extrusion, and thermoforming. Injection molding is a manufacturing process where melted polymer is forced into a mold cavity under pressure. A mold cavity is essentially a copy of the part being produced. The cavity is filled with plastic, and the plastic changes phase to a solid product. 
Typically, injection pressures range from 5000 to 30,000 psi. Because of the high pressures involved, the mold must be clamped shut during injection and cooling. The injection molding process is capable of producing large numbers of parts to very high levels of precision [2]. Injection molded thermoplastics are already used, for instance, in MEMS packaging as well as in 3D electronic circuits (Molded Interconnect Devices) [3]. One of the most common integration approaches is a system-in-foil application which has been known as a way to realize electronic systems. In this concept, a flexible polymer substrate is used as a base substrate where electronic components are assembled and then by using different manufacturing methods they can be encapsulated and formed to a product [4]. One possible way to directly integrate various functionalities into plastic products is the use of a conventional injection molding process to over-mold flexible electronic circuits [5]. Over-molding is a process of adding an additional layer of material over an already existing object. This process is regularly used to manufacture parts, sub-sections of parts, and for prototype development. Typically, the substrate material will be bonded and mechanically interlocked with other materials . This material is placed into the injection molding tool. Then the over-mold material is shot into or around the substrate which is in our case, a flexible foil with assembled electronic components. When the overmold materials solidify, the two materials become joined together as one single product. Over-molding varies according to the materials' choice, so if the substrate is metal and the over-molding is plastic, any type of thermoplastic can be used, but in the case of over-molding a plastic part with another plastic, then there can be some compatibility issues, which will be discussed in the following sections.

\section{Over-molded integrated foils process flow}

The concept of over-molding integration is based on a combination of flexible electronic substrates, electronic components assembly, film forming, injection molding, and in-mold processes. The idea is to assemble SMD components on flexible substrates or circuit boards, and use the former as an insert in a conventional injection molding machine [6]. In such a way a required encapsulation process is needed for the desired application. By using flexible plastic foils and costeffective printing methods together with overmolding process, there is a great potential for building a manufacturing technology platform that can provide products in various applications fields with complex shapes at low cost. This work focuses to implement test vehicles where electronic components are assembled on flexible copperpolyimide foils [7]. The process flow for flexible electronic foils is explained in detail :
1. Clean the copper surface \& micro etch copper.

2. Laminate dry film photoresist onto the substrate using dry film laminator. UV illuminate the photoresist.

3. Develop the photoresist.

4. Etch samples and use stripping to remove the resist from the patterned copper.

5. Apply an OSP to protect the patterned copper from oxidation.

6. The flex circuit is laser cut, thus cutting it into two parts and cut an opening for the polymer to flow during over-molding process.

7. Finally, components are assembled using a conventional lead-free solder. The whole process is shown in figure 1

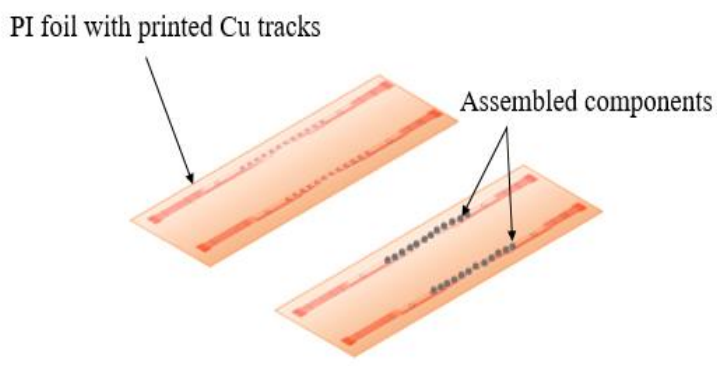

Figure 1: Integrated flexible foils process

The result is a flex foil with an assembled electronic circuit. This circuit is now prepared to an overmolding process, illustrated in fig. 2 .

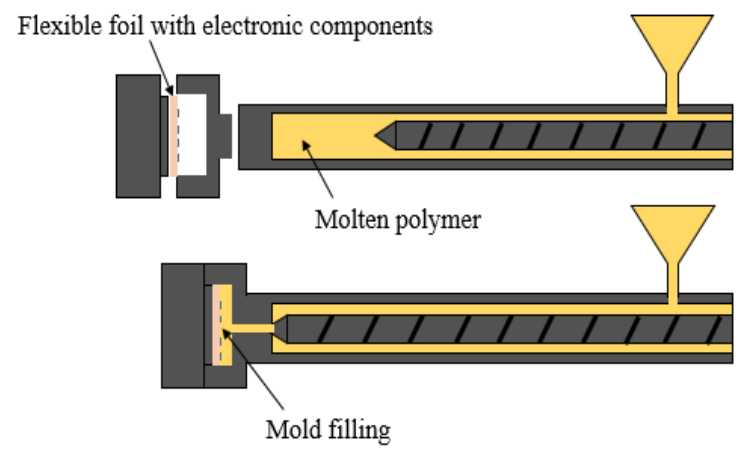

Figure 2: Schematic over-molding process

Before over-molding the desired polymer, the overmolded material (flexible foil with assembled components) should be dried in a convection oven to remove moisture, which can lead to expanding gas bubbles during the process. During over-molding as shown in fig. 2, the printed flexible foil in fig. 1 is clamped in the mold. The over-molding polymer is heated beyond its melting temperature. After the desired temperature is achieved, the mold is closed on the foil. Once the polymer is melted the pressure moves the injection screw to push the material towards the mold. The polymer starts to flow into 
cavity with the integrated foil, thus achieving the desired product shape in a very short time (less than a second). Finally when the temperature is sufficiently low and the over-molding polymer has solidified the mold opens again and the over-molded foil is ejected from the machine. The result is a dogbone shaped plastic with integrated resistors fig. 3 .

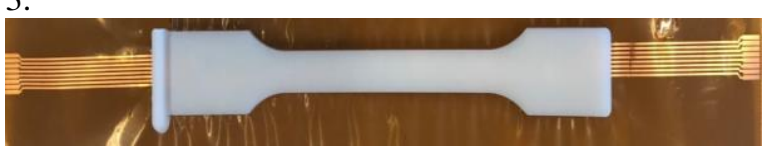

Figure 3: Over-molded sample with integrated resistors

\section{Adhesion between engineering thermoplastics and flexible foils}

Integration of flexible electronic circuits into engineering thermoplastics by over-molding technique can be realized in two approaches as schematically shown in fig.4: namely (1) encapsulating the flexible electronic circuits into engineering thermoplastic structure with undercut mold design (top), or (2) utilizing the adhesion between flexible electronic circuit foils and engineering thermoplastics to realize the structural integrity (bottom).

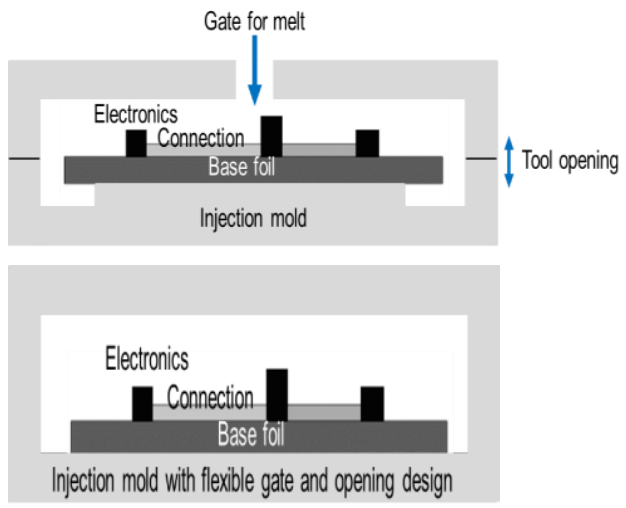

Figure 4: Mold design for encapsulating flexible electronic circuits (top) and mold design for adhering flexible electronic circuits with engineering thermoplastics (bottom)

The encapsulated flexible electronics can show a well-performed initial structural integrity with a wide range of base foil-engineering thermoplastic material combinations. However, if the adhesion between foil and thermoplastic material is not optimized, an undesired separation between base foil and engineering thermoplastic in long-term application may happen, which is inevitable due to the significant coefficient of thermal expansion (CTE) difference between typical foil material (neat polymer, CTE $\sim 10^{-5}$ ) and typical engineering thermoplastic (fiber filled polymer, CTE $\sim 10^{-6}$ ). The separation can either formulate wrinkles and bumps of flexible foil showing deteriorated aesthetic appearance or influence the joining between electronics and base foil/connection resulting damaged functionality of the electronics. In comparing with encapsulation design, the adhering design offers more flexibility in designing the mold. In addition, the optimized adhesion between foil and engineering thermoplastic can result in a muchimproved long-term structural integrity. Therefore, it is necessary to evaluate the adhesion performance between foils and engineering thermoplastics to screen the optimized material combination for overmolding. Fracture energy based peel test is used to evaluate adhesion between various types of foils and engineering thermoplastics. Since such test, as critically assessed, is capable to characterize fracture energy between dissimilar materials, which is independent of the geometry of test specimens, and mechanical property of materials [8]. In this study, the base foil (PI foil with $\mathrm{Cu}$ meanders) will be overmolded with different engineering plastics into the form of peel test specimens in order to check the best adhesion performance. The engineering thermoplastics used in this study are all commercialized materials, which are widely applied in automotive, and electronics industry. Four types of engineering thermoplastics are involved in this study Polypropylene (PP), 30\% weight percentage glass fiber filled polypropylene (GF-PP), Polyamide-6 (PA6) and 50\% weight percentage glass fiber filled polyamide-6 (GF-PA6). The injection molding parameters are in accordance with the recommendation from manufactures of engineering plastics, within the boundary, a relatively higher temperature is applied to promote adhesion. Injection molding melt temperature of 240 ${ }^{\circ} \mathrm{C}$ and mold temperature of $65^{\circ} \mathrm{C}$ is used for PP and GF-PP (with melting point $160{ }^{\circ} \mathrm{C}$ ), injection molding melt temperature of $270{ }^{\circ} \mathrm{C}$ and mold temperature of $80^{\circ} \mathrm{C}$ are employed for PA6 and GFPA6 (with melting point $220{ }^{\circ} \mathrm{C}$ ). As it was mentioned previously, in our case the PI foil with copper tracks is placed into the mold and the injection process starts where the polymer, with opposite equal flow directions, covers the electronic components (resistors) as shown in fig.5.

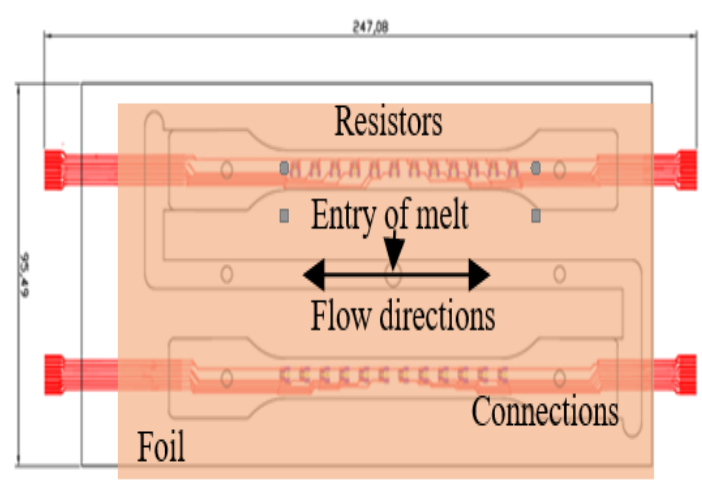

Figure 5: Schematic mold design 

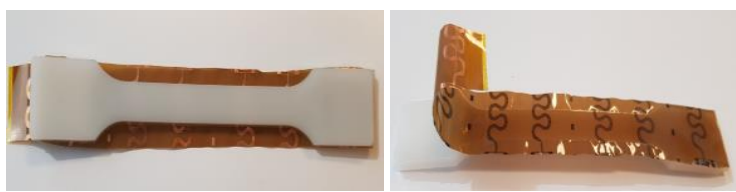

Figure 6: A trimmed over-molded flexible electronic circuit for peel test

The component will be further trimmed into the peel test specimen as shown in fig.6.The over-molded flexible electronics foil with PP is shown in fig.7. It can be observed that bonding between PP and foil is not completed. This is an expected observation, since the adhesion between dissimilar materials strongly relies on the formation of chemical bonds, and $\mathrm{PP}$ is lacking reactive chemical function groups.

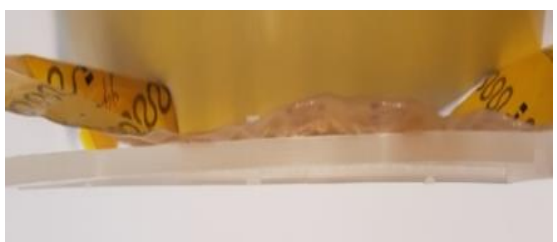

Figure 7: Over-molded flexible electronics foil with PP

Figure 8 shows the over-molded flexible electronics foil with GF-PP, which shows a comparable result with fig. 8.

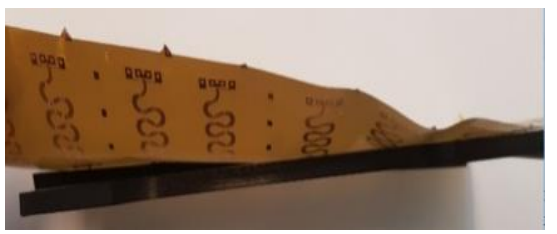

Figure 8: Over-molded flexible electronics foil with GF-PP.

Strong adhesion is observed between the foil and over-molded PA6 as shown in fig.9.This may be attributed to the high reactive $\mathrm{C}=\mathrm{O}$ bond in PA6 which promote chemical bonding with PI on the foil surface.

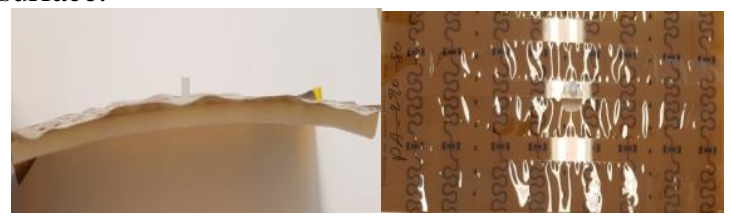

Figure 9: Over-molded flexible electronics foil with PA6.

Figure 10 shows the over-molded flexible electronics foil with GF-PA6. The adhesion between UPISEL-N foil and GF-PA6 is also weak with a large area of separation. The weak bonding between foil and GF-PA6 is not attributed to interfacial thermal residual strain energy, which is indeed lower in foil/GF-PA6 system than foil/PA6 system. Since the CTE of GF-PA6 $\left(10 \mathrm{ppm} /{ }^{\circ} \mathrm{C}\right)$ is more comparable to UPISEL-N foil $\left(18 \mathrm{ppm} /{ }^{\circ} \mathrm{C}\right)$ than the PA6 $\left(90 \mathrm{ppm} /{ }^{\circ} \mathrm{C}\right)$, this results in a much lower interfacial thermal residual strain energy tending to separate the interface. Anticipation on explaining this difference between PA6 and GF-PA6 is the effect of short glass fibers which may migrate to the interfacial region and partly embedded in foil during the over-molding process. As schematically illustrated in fig.11, since the adhesion between foil and glass fiber can hardly be realized, multiple microcracks may be initiated at the interfacial region, these cracks can propagate under a relatively low loading such as interfacial thermal residual stress.

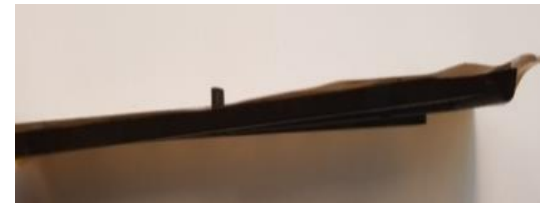

Figure 10: Over-molded flexible electronics foil with GF-PA6.

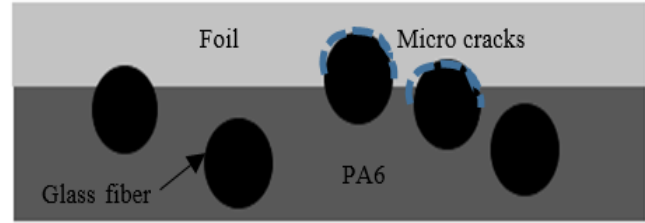

Figure 11: Schematically view of the interfacial region of the foil/GF-PA6 system

As a summary of this material screening test, a strong bonding is performed in the foil-PA6 system while the other material combinations hardly realize measurable adhesion. Therefore, PA6 material is employed in the following functionality test for its best adhesion performance.

\section{Test Vehicle Description}

To check the performance of the whole mechanism, experiments on the integrated foils during the over-molding process have been performed. A test vehicle realized on samples of PI$\mathrm{Cu}$ foils are used to allow electrical testing of the embedded components by using contact pads on the flex foil. Each test sample has a total length of $247.08 \mathrm{~mm}$ and a width of $95.49 \mathrm{~mm}$. Test structures had 24 pieces of SMD $(0 \mathrm{ohm})$ resistors in 0402 packages. All SMD components were assembled using lead-free solder. Fig.12 shows the sample used in the test vehicle.

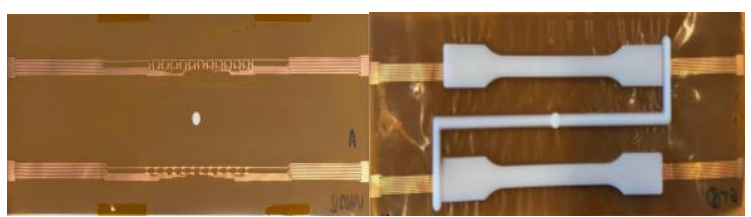

Figure 12: Sample before (left) and after (right) OVM 
Test samples were studied in several conditions; without underfill, with underfill as well as with both underfill and glob top application. These conditions were compared in order to make an overall comparison for the test vehicles. Moreover, the overmolding process tested for two different cycles to check the integrity of the samples in different temperature and pressure conditions. Table1 includes the conditions of the test vehicle. Furthermore, all test vehicles performed on two types of polyimide (PI)/Copper $(\mathrm{Cu})$ tracks foils with and without an adhesive layer between PI and $\mathrm{Cu}$.

Table 1: Injection molding process parameters

\begin{tabular}{|l|l|l|}
\hline Parameter & Cycle 1 & Cycle 2 \\
\hline Material Temperature & $250{ }^{\circ} \mathrm{C}$ & $270{ }^{\circ} \mathrm{C}$ \\
\hline Mold Temperature & $50{ }^{\circ} \mathrm{C}$ & $80{ }^{\circ} \mathrm{C}$ \\
\hline Pressure & $562 \mathrm{bar}$ & $451 \mathrm{bar}$ \\
\hline Injection time & $0.22 \mathrm{sec}$ & $0.46 \mathrm{sec}$ \\
\hline Holding time & $25 \mathrm{sec}$ & $25 \mathrm{sec}$ \\
\hline Cooling time & $25 \mathrm{sec}$ & $25 \mathrm{sec}$ \\
\hline
\end{tabular}

\section{Electrical measurements before and after OVM}

The measurement of the test samples is done pre and post over-molding by using a twopoint resistance measurement system. Which means that a multimeter is connected by two probes to the contacting pads on the foil as shown in figure 13 .

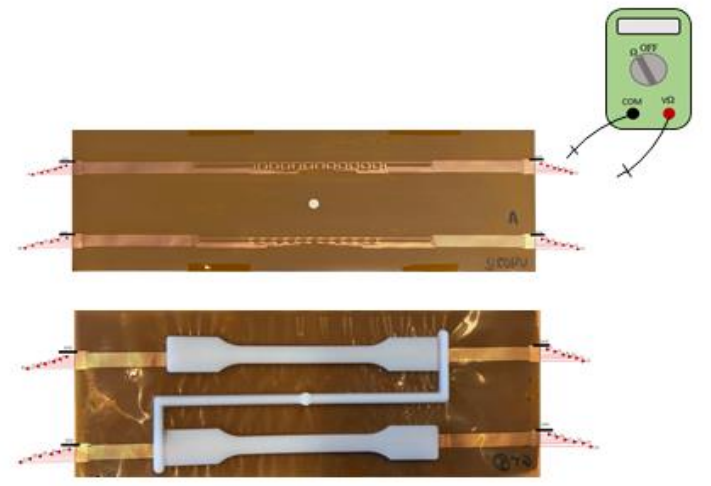

Figure 13: Two-probe measurement

Each test sample has four grounds marked in black like in fig. 13 while the rest are connection lines numbered according to the resistor location. In this case, we have 24 resistance readings ( one reading for each of the individual resistors) for each sample. Our aim is to check whether the components are still functional or not after over-molding process, thus the resistance values should not change after overmolding.

\section{Results and discussion}

It was shown that in figure 15 (cycle 2) the sample exposed to high temperature environment gave better results in adhesion and in the connection performance. Because higher temperature means reduced viscosity of the liquid polymer, less injection pressure and accordingly less mechanical stress on the components. Also the resistance values were almost the same and did not get higher while in fig. 14 (cycle 1) the resistance values after overmolding became higher due to the higher mechanical stress on the components. Moreover, all components were still functional after over-molding process. Samples were visually inspected and no sample damages, component damages or displacement or other evident defects were observed.

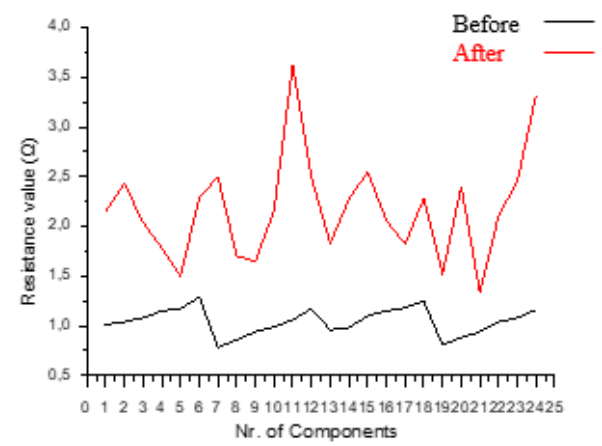

Figure 14: Measured resistance values before and after OVM at low temperature (cycle 1)

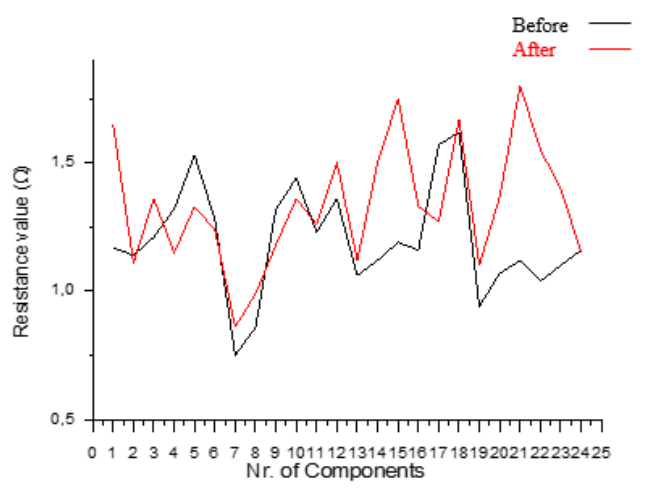

Figure 15: Measured resistance values before and after OVM at high temperature (cycle 2)

It was shown that UPISEL-N (without adhesive layer) polyimide foil is more compatible with overmolding process conditions. As shown in fig.16, the PI foil with adhesive layer showed delamination in the $\mathrm{Cu}$ tracks that lead to an open circuit in the connection post over-molding and sometimes the sample was completely destroyed during the overmolding process. For these reasons, all tests were performed on UPISEL-N foil. 


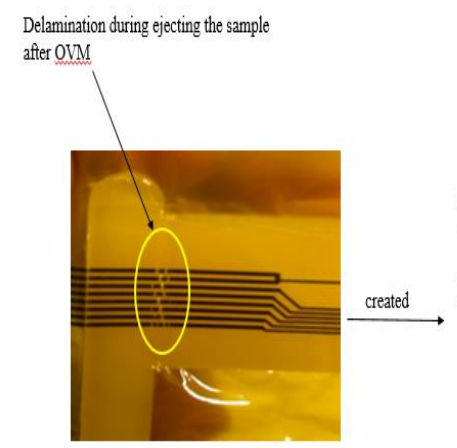

Complete foil deformation after OVM

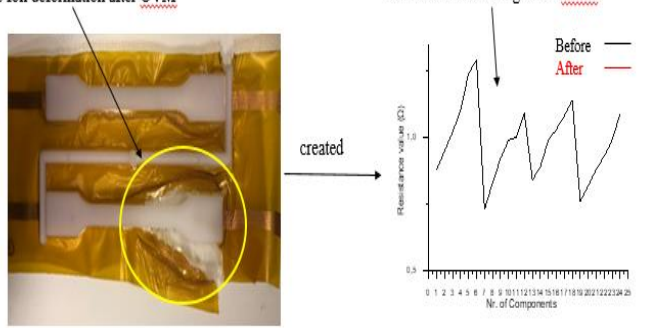

Figure 16: Failures in PI foil with an adhesive layer

It was shown that the presence of an underfill material below the components has a contribution to the measurements. In fig.17, some samples were over-molded without an underfill material, resulting in high resistance values than pre-over-molding readings. But, when adding underfill material as shown in fig.18, The resistance values become more stable compared to the readings in fig. 17 after overmolding.
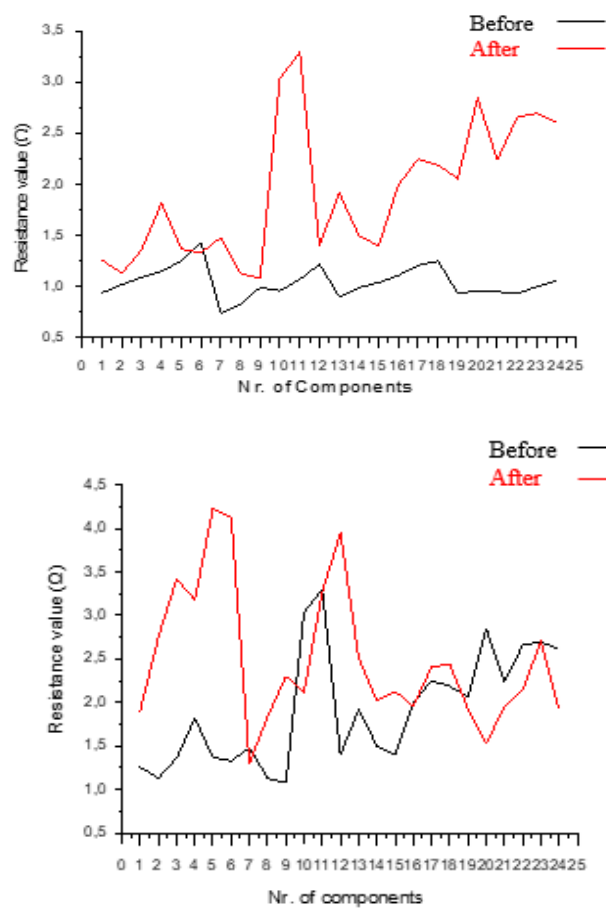

Figure 17: OVM without underfill material at $250{ }^{\circ} \mathrm{C}$
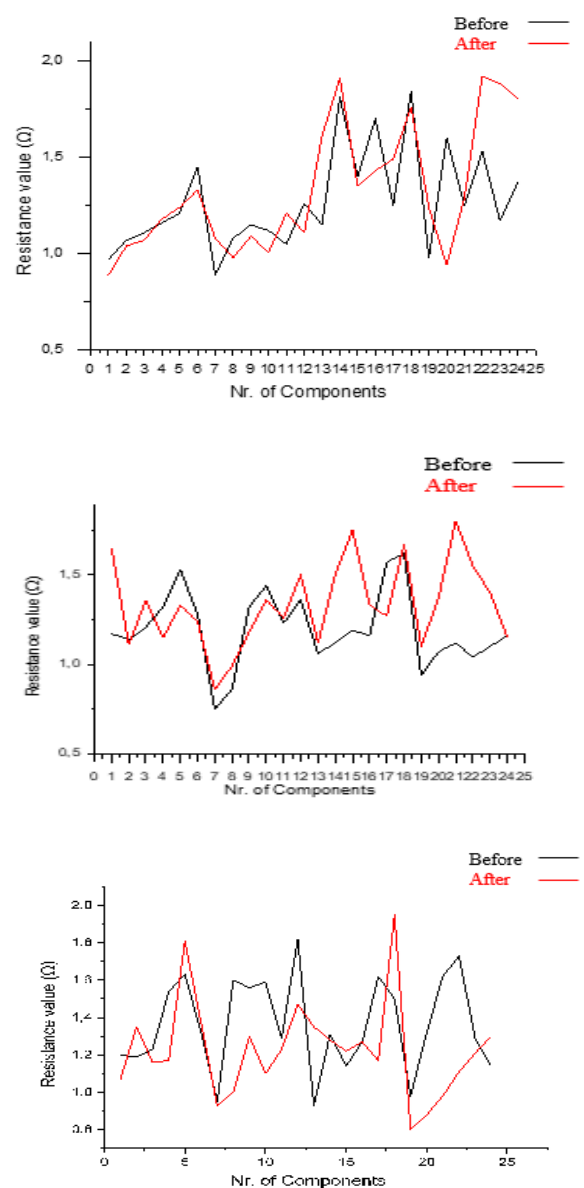

Figure 18: OVM with underfill material at 270 ${ }^{\circ} \mathrm{C}$

Furthermore, the influence of glob top material was studied as well. Some components were surrounded with epoxy-based material and their resistance values are shown in fig. 19 , dried at $100{ }^{\circ} \mathrm{C}$ for 15 minutes resulting in slightly lower resistance values compared to fig.16 which was for components with underfill material below them with no glob top.
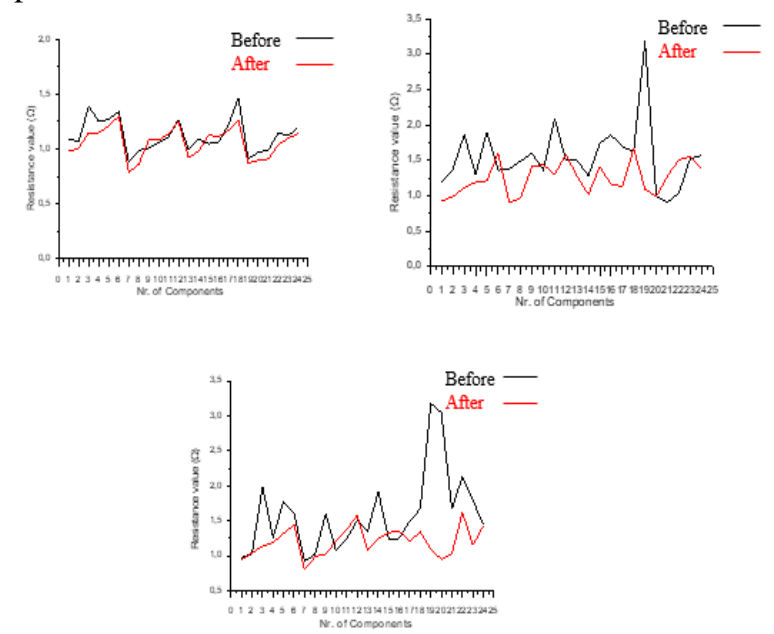

Figure 19: OVM of samples with glob top and underfill application at $270{ }^{\circ} \mathrm{C}$ 


\section{Simulation of OVM process}

In order to understand the effect of injection molding process condition on the functionality of electronic components, the entire OVM process is simulated by Moldex3D software. Figure 20 shows the model established in Moldex3D environment, which excludes copper connection due to the feature of thin layer, small area and high thermal conductivity.

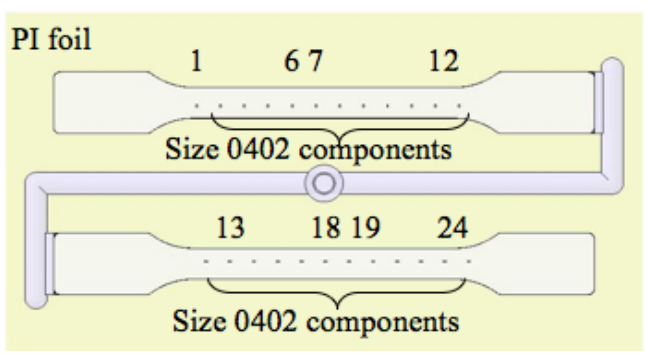

Figure 20: Model of simulation

The previously mentioned OVM cycle 2 is simulated and the temperature history of selected components from figure 20 is shown in figure 21 . The simulation shows that the temperature of components drops from $250^{\circ} \mathrm{C}$ to $220{ }^{\circ} \mathrm{C}$ in 5 seconds, which finally reaches $120{ }^{\circ} \mathrm{C}$ in the end of cycle. Therefore, the soldering (melting point of $260{ }^{\circ} \mathrm{C}$ ) under OVM cycle 2 (injection temperature of $270{ }^{\circ} \mathrm{C}$ ) is not melted thus the functionality of resistors is possible to be retained.

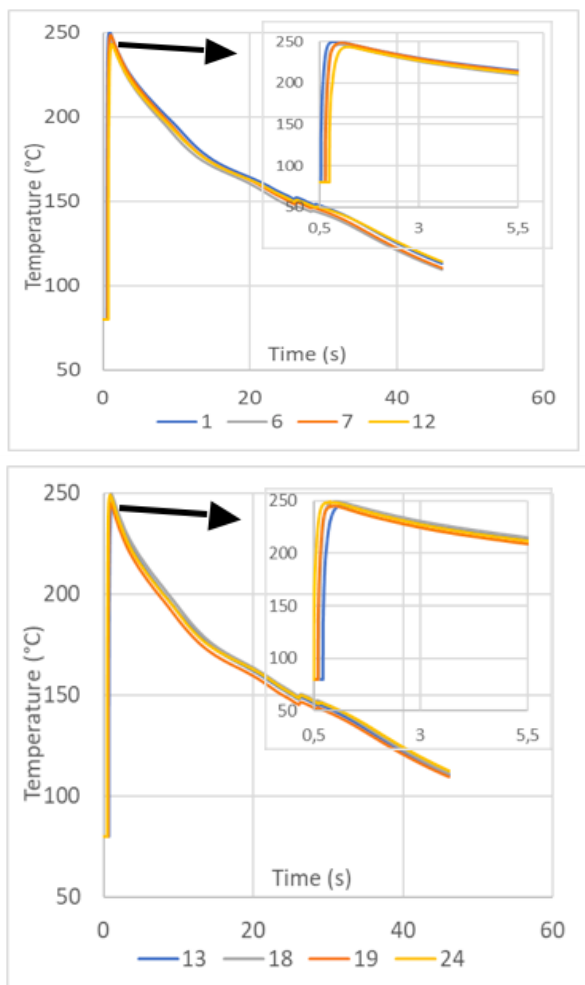

Figure 21: Temperature history of selected components

\section{Conclusions}

The technology presented in this paper proves that over-molding integration could be a feasible technology enabling an ideal integration of electrical features into $3 \mathrm{D}$ plastic products. It also shows that electrical functionality can be integrated efficiently inside the thermoplastic polymer. It also proves that the number of the embedded SMD components can be relatively high and still functional under pressure and temperature of the over-molding process.Underfill material showed better rsults, at least for the component and designs used in this study. However, the change in resistance was still within the measurement error of the two probe measurement method. Performing the overmolding in high temperature condition is essential for better adhesion results. PA6 showed the best adhesion compared to other materials discussed in this paper. Application of glob top is not yet confirmed whether or not it had a major influence but further experiments will be performed to clarify this point. Furthermore, different electronics packages such as capacitive touch, sensors, micro controllers and LEDs will also be a topic for further study.

\section{Acknowledgments}

This work is financed through the Flexlines project within the Interreg V-programme FlandersThe Netherlands, a cross-border cooperation programme with financial support from the European Regional Development Fund, and cofinanced by the Province of East-Flanders, Belgium.

\section{References}

[1] Gonzalez et al, "Design and implementation of flexible and stretchable systems", Microelectronics Reliability, Vol.51, No.6, pp.1069-1076, March 2011.

[2] Sina Ebnesajjad, "Fluoroplastics", William Andrew Publishers, second edition, Volume 2, pp. 151-193, 2003.

[3] K. Gilleo, D. Jones, G. Pham-Van-Diep, "Thermoplastic Injection Molding: New Packages and 3D Circuits", Proceedings of the 10 ECWC Conference at IPC Printed Circuits Expo, 2005.

[4] M. Koyuncu, "Systems Integration: From Embedded Components to Integrated Flexible Systems", Proceedings of the Plastic Electronic Conference, Dresden, Germany, October 19- 21st, 2010. 
[5] T. Alajoki et al.,"Hybrid in-mould integration for novel electrical and optical features in 3D plastic products," Proceedings of the 4th Electronic SystemIntegration Technology Conference, Amsterdam, Netherlands, pp. 1-6, 2012.

[6] N.J.Teh et al., "Embedding of Electronics within Thermoplastics Polymers by Injection Moulding", Proceedings of the IEEE/CPMT International Electronics Manufacturing Technology Symposium, pp. 10-18, 2000.

[7] B. Plovie et al.,"One-time deformable thermoplastic devices based on flexible circuit board technology," Proceedings of the 11th International Microsystems, Packaging, Assembly and Circuits Technology Conference (IMPACT), Taipei, pp.125-128,2016.

[8] Y. Su, M. deRooij, W. Grouve, L. Warnet, "Characterisation of metal-thermoplastic composite hybrid joints by means of a mandrel peel test", Composites Part B: Engineering, Vol. 95, pp. 293-300, June, 2016. 